\title{
SIMULAÇÃO DE COMBUSTÃo DO POLIBUTADIENO LÍQUIDO HIDROXILADO, PERCLORATO DE AMÔNIO E ALUMÍNIO EM FORMULAÇÕES DE PROPELENTE SÓLIDO COMPÓSITO
}

\author{
Renata F. Cardoso ${ }^{\text {a }}$, Luciene D. Villar ${ }^{\text {, }}$, Elizabete Y. Kawachi ${ }^{\mathrm{a}}$ e Rene F. B. Gonçalves ${ }^{\mathrm{a}, *,(1)}$ \\ aDepartamento de Química, Divisão de Ciências Fundamentais, Instituto Tecnológico de Aeronáutica, 12228-900 São José dos \\ Campos - SP, Brasil \\ bDepartamento de Ciência e Tecnologia Aeroespacial, Instituto de Aeronáutica e Espaço, 12228-900 São José dos Campos - SP, Brasil
}

Recebido em 31/08/2018; aceito em 06/12/2018; publicado na web em 16/01/2019

\begin{abstract}
COMBUSTION SIMULATION OF HYDROXYL-TERMINATED LIQUID POLYBUTADIENE, AMMONIUM PERCHLORATE AND ALUMINUM IN COMPOSITE SOLID PROPELLANT FORMULATIONS. The present work presents combustion simulations of hydroxyl-terminated liquid polybutadiene loaded with ammonium perchlorate and aluminum, using Chemkin software and the "Plug Flow" type reactor. These materials are widely used in solid composite propellant formulations. Through this study, the effect of parameters of axial velocity, equivalence ratio, pressure and temperature during the firing of the propellant were discussed. The objective of this work was to discuss how to define the best parameter value based on the obtained results, and thus generate optimized formulations.
\end{abstract}

Keywords: combustion; composite solid propellant; kinetic simulations.

\section{INTRODUÇÃO}

Um motor foguete movido à propulsão química consiste basicamente em uma câmara cilíndrica, na qual ocorre uma reação de combustão, gerando grandes quantidades de gases em altas temperaturas. Estes gases, ao serem expelidos pela tubeira, um estreitamento do motor foguete, provocam propulsão no sentido oposto. A propulsão do foguete é responsável pela geração de energia que o move, e é originária da queima de um par combustível-oxidante, comumente chamado de propelente.

Os motores foguete à propulsão sólida utilizam um tipo especial de propelente sólido denominado propelente sólido compósito (PSC) para lançamento de foguetes orbitais e suborbitais. Trata-se de um compósito polimérico de formulação complexa, tendo como fase contínua uma matriz polimérica, e por fase descontínua um sal inorgânico oxidante, rico em oxigênio, e um aditivo metálico, para aumentar a temperatura dos gases de combustão e, consequentemente, a eficiência energética do motor foguete.

No Brasil, a formulação de propelentes para aplicação em foguetes de sondagem e lançador de satélites, como o VLS-1, utilizam a resina polibutadiênica hidroxilada e o perclorato de amônio como oxidante. A resina compõe uma parte importante do propelente, como a matriz polimérica, a qual atua como ligante, em inglês: binder, aglutinando a carga sólida para formar um material coeso e com resistência mecânica suficiente para suportar os esforços aos quais o propelente está submetido durante as etapas de manuseio, transporte, armazenamento, ignição e voo do motor foguete. A queima deste tipo de propelente produz um elevado volume de gases e chega à uma temperatura de $3000 \mathrm{~K}$, características que são essenciais para que o processo de combustão produza energia necessária ao voo do foguete.

Devido à dificuldade de realizar experimentos com estes materiais em condições reais de combustão, as técnicas de modelagem computacional são aplicadas para estimar o comportamento da queima de propelentes sólidos. O Chemkin é um software muito utilizado para estudar a chama formada pela combustão de materiais

*e-mail: renefbg@ita.br energéticos, como o propelente. Este software considera as equações de conservação de massa e energia das espécies envolvidas, e o momento linear. Com isso, é possível analisar a temperatura e frações molares das espécies, em função da distância de queima do material na superfície.

O presente trabalho aborda sobre a simulação da combustão da mistura PBLH/Perclorato de Amônio/Alumínio, utilizadas em propelente sólido compósito, focando na temperatura de chama, bem como na formação e decomposição das espécies químicas envolvidas. Os estudos foram realizados variando-se a velocidade axial, razão de equivalência, pressão e temperatura de queima inicial. A escolha de cada condição ideal foi conduzida avaliando o perfil de chama, produtos de combustão e temperatura e pressão máxima obtida em cada simulação.

\section{Propelente Sólido Compósito (PSC)}

É uma mistura complexa e estável que consiste em duas fases: oxidante/redutor (percloratos, nitratos/ alumínio) dispersos em um ligante combustível, como um elastômero de poliuretano (PU). Suas principais aplicações estão relacionadas à propulsão química por motor foguete, mísseis e projéteis de armamentos. O motor-foguete, Figura 1, apresenta o envelope motor, que é fabricado com material metálico ou compósito de alta resistência, possuindo uma proteção térmica, a qual é responsável pela barreira térmica e proteção do envelope motor, o ignitor que é o iniciador da queima do propelente, a câmara de combustão, onde ocorre o aumento de espécies gasosas com grande elevação da temperatura e pressão, a tubeira, que é onde saem os gases provenientes da queima do propelente. . $^{3,6}$

As formulações de propelentes sólidos podem ser descritas como elastômeros altamente carregados, geralmente um poliuretano, em que a matriz polimérica (binder) irá envolver as partículas sólidas que compõem a formulação do combustível sólido, promovendo propriedades mecânicas de tal forma que o grão resultante não sofra nenhuma falha de comportamento mecânico durante a fabricação, transporte, manuseio e operação do motor. O binder também atua como fonte de carbono no processo de queima. ${ }^{2}$, A Figura 1 apresenta o esquema simplificado das partes do motor foguete à propulsão sólida. 


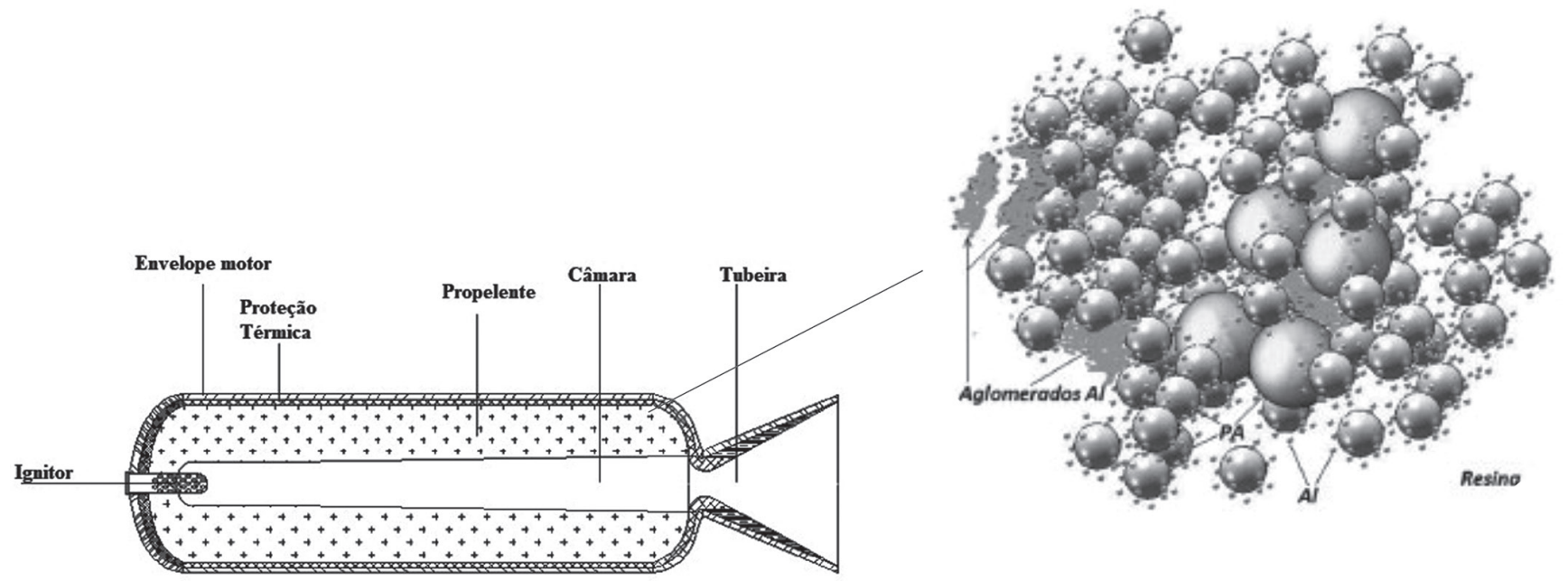

Figura 1. Esquema simplificado de um motor foguete. ${ }^{6,8}$

Entretanto, diferentemente de outros compósitos, o PSC apresenta como componente em maior concentração a carga sólida, podendo variar desde 60 até $80 \%$ em concentração mássica de um sal oxidante, além de um aditivo metálico (10 a 15\%). Esses sólidos conferem ao propelente suas características de material energético. A Tabela 1 apresenta a composição de uma formulação típica de PSC.

Tabela 1. Formulação típica de propelente sólido compósito ${ }^{2}$

\begin{tabular}{lc}
\hline Componente & Concentração $(\mathrm{m} / \mathrm{m} \%)$ \\
\hline Aglutinante polimérico & 10 a 15 \\
Oxidante & 60 a 80 \\
Aditivo metálico & 10 a 15 \\
Agente de cura & Variável em função da razão molar $[\mathrm{NCO} /[\mathrm{OH}]$ \\
Aditivos & $<5$ \\
\hline
\end{tabular}

Dentre os oxidantes utilizados, destaca-se o perclorato de amônio, como aditivo metálico, o alumínio em pó e, como aglutinante, destaca-se o polibutadieno hidroxilado (PBLH), cuja designação em inglês é dada por hydroxyl-terminated polybutadiene (HTPB).

Propelentes compósitos contendo oxidantes cristalinos, como perclorato de amônio e hidrocarbonetos poliméricos, também queimam para gerar fragmentos de oxidante e combustível por decomposição térmica. Esses fragmentos se difundem entre si para formar um gás pré-misturado na superfície de combustão e reagir para formar produtos de combustão de alta temperatura.

\section{Poliol}

O termo poliol refere-se a uma classe de compostos orgânicos que possuem grupos hidroxilas $(\mathrm{OH})$ em sua estrutura, as quais podem reagir com o grupo isocianato $(\mathrm{NCO})$. As resinas são utilizadas para sintetizar o poliuretano (PU), que é a matriz polimérica do propelente. O polibutadieno líquido hidroxilado (PBLH) é comumente utilizado nas formulações de propelente devido às vantagens: alta elasticidade e propriedades mecânicas adequadas à aplicação em propelente compósito, resistência à hidrólise, suporta altos carregamentos de materiais particulados sem alterar significativamente suas propriedades mecânicas originais., O PBLH é sintetizado através da reação de polimerização do butadieno, em presença de peróxido, utilizando um álcool como diluente. Através deste método, o PBLH possui isômeros com composição de $20 \%$ da estrutura cis, $60 \%$ da trans e $20 \%$ da vinil em sua cadeia hidrocarbônica (Figura 2) e suas moléculas são ligadas entre si por meio de ligações químicas covalentes, conferindo ao poliuretano uma alta elasticidade e propriedades mecânicas adequadas à aplicação em propelente compósito. ${ }^{11}$,

O polibutadieno líquido hidroxilado (PBLH) é obtido através da reação de polimerização via radicais livres. A reação constitui-se na utilização do peróxido de hidrogênio como iniciador da reação de polimerização do monômero de butadieno, obtendo-se um polibutadieno difuncional com hidroxilas terminais do tipo alílica, bastante reativas.

\section{Perclorato de amônio}

O perclorato de amônio (AP), Figura 3, é utilizado como oxidante em formulações de propelente. O AP é um sólido cristalino, de estrutura ortorrômbica, que pode mudar para cúbica quando atinge uma temperatura de $513 \mathrm{~K}$, mudança que pode afetar o processo de decomposição. É um oxidante não higroscópico na atmosfera, apresenta fração de massa de oxigênio igual a 0,545 e é relativamente estável em choques mecânicos. ${ }^{11}$

\section{Alumínio}

O alumínio é utilizado como aditivo metálico em formulações de

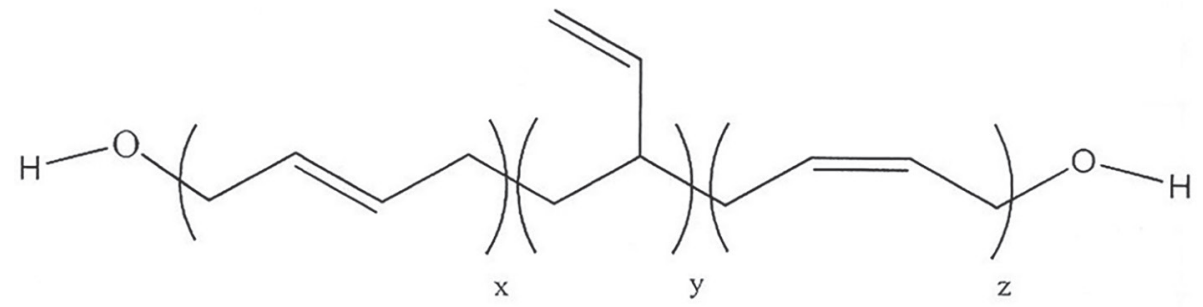

Figura 2. Estrutura química do PBLH. Isômeros representados por: $x$, trans(60\%); y, vinil (20\%); $z$, cis (20\%) 


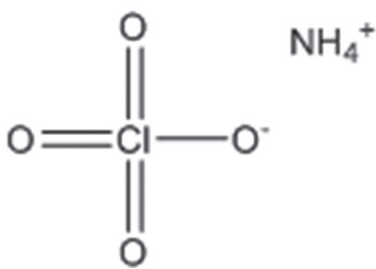

Figura 3. Estrutura do perclorato de amônio

propelente sólido compósito. O alumínio tem a função de estabilizar o processo de queima contra as instabilidades, além de aumentar a energia envolvida durante a combustão. ${ }^{5,11}$

\section{Produtos de combustão}

O perclorato de amônio (AP) produz fragmentos ricos em oxidantes, porém a sua densidade de energia não é alta, assim como o aglutinante polimérico. A densidade de energia faz referência à quantidade de energia desprendida durante a combustão do composto. No caso do perclorato de amônio, a energia liberada por unidade de massa durante sua decomposição não atinge valores considerados altos, porém disponibiliza oxigênio necessário para a combustão do binder e dos outros componentes presentes. Entretanto, quando as partículas de AP e da matriz polimérica são misturadas, o material estequiometricamente equilibrado, denominado propulsor compósito AP, é formado. Com o objetivo de aumentar o impulso específico, o alumínio em pó é adicionado como um componente combustível. Devido aos componentes do propelente, durante a queima é possível visualizar uma fumaça branca na saída da tubeira do foguete. Estes produtos de combustão são majoritariamente: ácido clorídrico $(\mathrm{HCl})$, óxido de alumínio $\left(\mathrm{Al}_{2} \mathrm{O}_{3}\right)$, dióxido de carbono $\left(\mathrm{CO}_{2}\right)$, e água $\left(\mathrm{H}_{2} \mathrm{O}\right)$. $\mathrm{O} \mathrm{HCl}$ é o componente que gera a fumaça branca quando combinado com a água (mistura) na atmosfera. Quando o PBLH e o AP são decompostos termicamente na superfície do propelente, são formados gases provenientes do oxidante e combustível, que difundem entre si e reagem para produzir gases de combustão de alta temperatura, a qual pode atingir valores de $3000 \mathrm{~K}$ e pressão na faixa de 20 à $70 \mathrm{~atm}$. $^{4,11}$,

O mecanismo proposto por Chiung-Chu e McQuaid, mesmo sem diferenças significativas entre os produtos de combustão, considera a espécie $\mathrm{NH}_{4} \mathrm{ClO}_{4}$ como produto de decomposição ao invés de $\mathrm{NH}_{3}+\mathrm{HClO}_{4}$, devido o $\mathrm{NH}_{4} \mathrm{ClO}_{4}$ ser mais semelhante com a fase condensada. Já para a resina PBLH, considerou-se como subproduto de pirólise, o hidrocarboneto $\mathrm{C}_{20} \mathrm{H}_{32}$, com a mesma proporção de butadieno cis/trans/vinil, a qual foi nomeada como R45M.

Os processos físico-químicos que ocorrem durante a combustão do propelente à base de PBLH/AP incluem aquecimento em fase condensada, degradação das espécies, fusão e pirólise superficial e reações em fase gasosa. As estruturas de chama e o comportamento de queima dependem de vários fatores, como composição do propelente, condições iniciais e ambientais e configuração morfológica do propelente. ${ }^{15}$

\section{Modelagem Chemkin}

O Chemkin, anacronismo de Chemical Kinetics, é um software que possui bancos de dados e sub-rotinas escritas em arquivos texto e em código FORTRAN para resolver problemas envolvendo cinética de fase gasosa, equilíbrio e propriedades de transporte. Existem diferentes problemas que podem ser resolvidos usando as sub-rotinas do Chemkin, alguns deles são: frações molares das espécies em função do tempo, velocidade de chama, frações molares das espécies e perfil de temperatura em função do tempo em chamas laminares, em estado estacionário e pré-misturadas, entre outros. Para isso, é possível escolher o melhor modelo de reator que se aplica ao experimento.

Um dos modelos que pode ser utilizado é o modelo chamado "Plug-Flow Reactor" (PFR). O PFR é um ótimo reator para estudar cinética química em temperaturas superiores a $1000 \mathrm{~K}$ e alta velocidade, o qual utiliza aproximações matemáticas para reatores de laboratório correspondentes. Além da aproximação com modelos de reatores práticos, o PFR também pode ser utilizado na modelagem de situações práticas complexas. Esse reator é utilizado em situações onde há um fluxo constante de gases reagentes em um canal ou duto, onde a seção transversal possui área fixa. Embora as espécies e a temperatura possam variar ao longo do comprimento do canal, considera-se que não haja variações no canal e não haja transporte difusivo ao longo do comprimento do canal, ou seja, na direção do fluxo. Os modelos PFR descrevem o reator de fluxo de tubo em estado estacionário, o qual pode ser utilizado para projeto, otimização e controle de processo. ${ }^{1} \mathrm{~A}$ análise é baseada em equações de conservação nas fases gasosas e condensadas, cinética química de taxa finita e propriedades termofísicas variáveis.

\section{PARTE EXPERIMENTAL}

A simulação foi conduzida pelo software Chemkin, no reator tipo "Plug-Flow". ${ }^{19} \mathrm{O}$ mecanismo de reação, bem como as propriedades de fase condensada, cinética química e parâmetros de transporte do mecanismo foram obtidos através do trabalho de ChiungChu e McQuaid. ${ }^{16}$ Os estudos realizados foram divididos em quatro condições, conforme Tabela 2, onde variou-se a velocidade axial (Estudo 1), composição da mistura oxidante/combustível (Estudo 2), pressão (Estudo 3) e temperatura (Estudo 4). Os dados da Tabela 2 foram utilizados como informações de entrada para cada simulação no software Chemkin-II. A escolha de cada condição otimizada foi realizada de acordo com a temperatura máxima, produtos de combustão completa e também quantidade de produtos de combustão incompleta formados durante a decomposição do propelente.

As condições de cada estudo estão na Tabela 3. Foram escolhidos seis pontos mais relevantes de cada estudo.

\section{RESULTADOS E DISCUSSÃO}

\section{Estudo 1 - Efeito da velocidade axial}

No estudo 1 foi conduzido um estudo paramétrico de velocidade axial, conforme Tabela 3. Os resultados comparativos das curvas de temperatura, em função da distância, e hidrocarbonetos não queimados em função da distância se encontram na Figura 4 e 5 , respectivamente. A temperatura que é expressa no gráfico é a temperatura atingida dentro da câmara de combustão durante a queima do propelente, e a distância é referente ao comprimento da câmara de combustão até a tubeira do motor-foguete.

Com os estudos de temperatura em função da distância, Figura 4, obtém-se informações sobre o perfil da chama, ou seja, é possível visualizar o comportamento da chama durante a queima do propelente. Além disso, nota-se a presença de regiões "frias", a qual a temperatura é mais baixa, resultando em uma diminuição considerável na velocidade das reações químicas. Sendo assim, o perfil de temperatura escolhido para ser utilizado nos estudos de outras variáveis foi o que teve a menor distância de região "fria" e maior velocidade de reação. Na Figura 4, todas as condições de velocidade axial estudadas apresentaram uma temperatura máxima de aproximadamente $2900 \mathrm{~K}$. Entretanto, os perfis ideais de chama foram obtidos nas condições 1 e 2, Figuras $4 \mathrm{a}$ e 4b, respectivamente, 
Tabela 2. Descrição das variáveis dos estudos realizados

\begin{tabular}{|c|c|c|c|c|}
\hline Condições de estudo & Estudo 1 & Estudo 2 & Estudo 3 & Estudo 4 \\
\hline Posição axial final $(\mathrm{cm})$ & 100 & 100 & 100 & 100 \\
\hline Diâmetro (cm) & 10 & 10 & 10 & 10 \\
\hline Temperatura $(\mathrm{K})$ & 1200 & 1200 & 1200 & Estudo paramétrico ${ }^{d}$ \\
\hline Pressão (atm) & 1 & 1 & Estudo paramétrico ${ }^{c}$ & 65 \\
\hline Velocidade axial $(\mathrm{cm} / \mathrm{s})$ & Estudo paramétrico $^{\mathrm{a}}$ & $10^{7}$ & $10^{7}$ & $10^{7}$ \\
\hline Razão de Equivalência & 1 & Estudo paramétrico $^{\mathrm{b}}$ & 1 & 1 \\
\hline Produtos de combustão & $\mathrm{Al}_{2} \mathrm{O}_{3}, \mathrm{HCl}, \mathrm{CO}_{2}, \mathrm{H}_{2} \mathrm{O}, \mathrm{N}_{2}$ & & & \\
\hline
\end{tabular}

${ }^{\mathrm{a}}$ Estudo 1: $10^{6}$ à 10 ${ }^{8}$; ${ }^{\mathrm{b}}$ Estudo 2: 0.05 à 1.0; ${ }^{\mathrm{c}}$ Estudo 3: 1 à $70 \mathrm{~atm} ;{ }^{\mathrm{d}}$ Estudo 4: 1200 à $2000 \mathrm{~atm}$

Tabela 3. Descrição dos estudos paramétricos em todas as condições

\begin{tabular}{|c|c|c|c|c|}
\hline \multicolumn{5}{|c|}{ Estudo Paramétrico } \\
\hline Condição & Estudo 1 - Velocidade Axial (cm/s) & Estudo 2 - Razão de equivalência & Estudo 3 - Pressão (atm) & Estudo 4 - Temperatura (K) \\
\hline 1 & $10^{6}$ & 0.05 & 1 & 1200 \\
\hline 2 & $10^{7}$ & 0.25 & 30 & 1378 \\
\hline 3 & $2.10^{7}$ & 0.50 & 46 & 1467 \\
\hline 4 & 5. $10^{7}$ & 0.75 & 55 & 1644 \\
\hline 5 & 7. $10^{7}$ & 1.0 & 65 & 1822 \\
\hline 6 & $10^{8}$ & 1.4 & 70 & 2000 \\
\hline
\end{tabular}

a) Velocidade axial - Condição 1

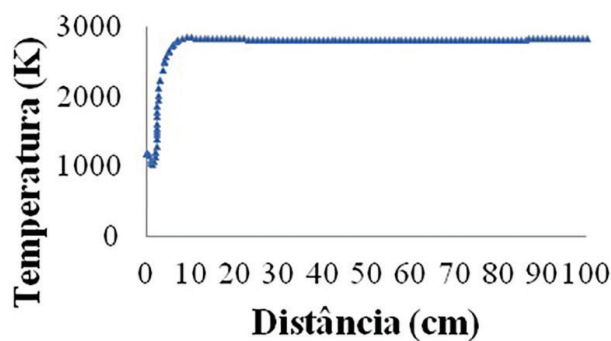

c) Velocida de axial - Condlição 3

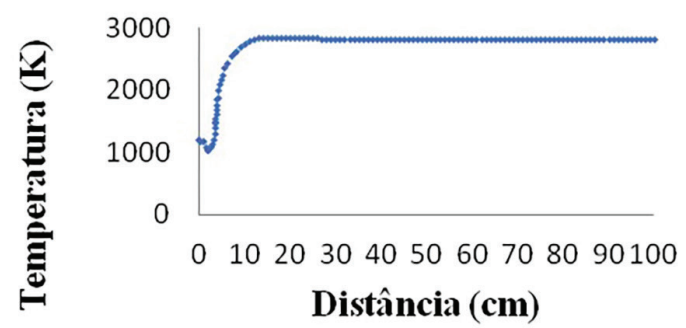

e) Velocidlade axial - Condição 5

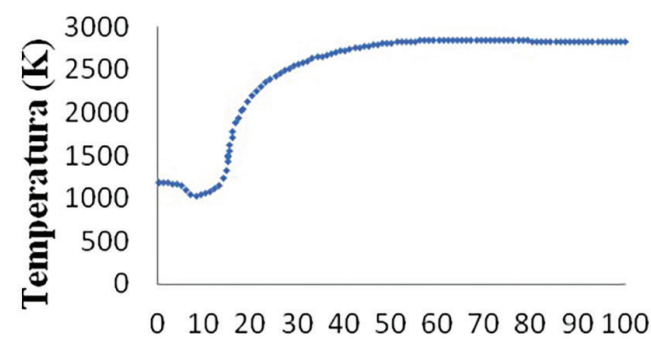

Distância (cm) b) Velocida dle axial - Condição 2

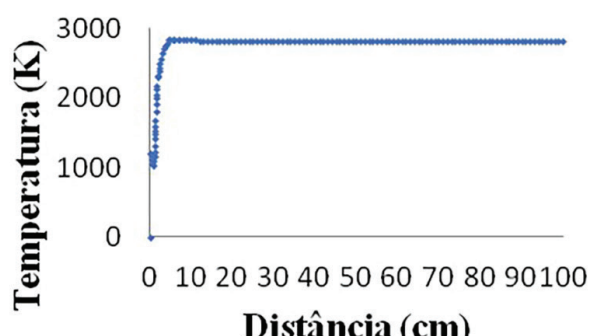

d) Velocidade axial - Condição 4

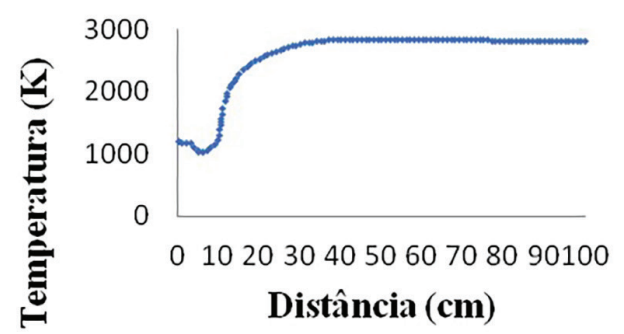

f) Velocidade axial - Condição 6

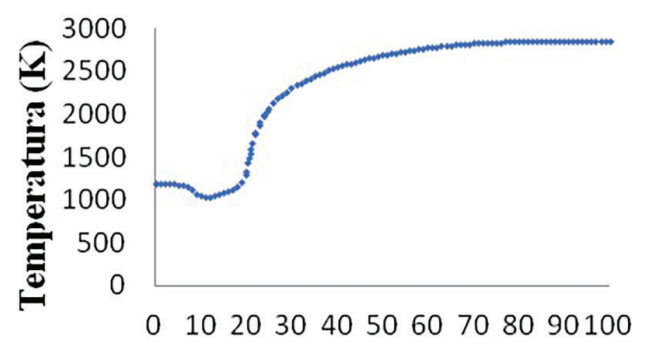

Distância (cm)

Figura 4. Perfil comparativo de temperatura em função da distância em cada condição de velocidade axial. a) Condição 1, b) Condição 2, c) Condição 3, d) Condição 4, e) Condição 5, f) Condição 6 
a)

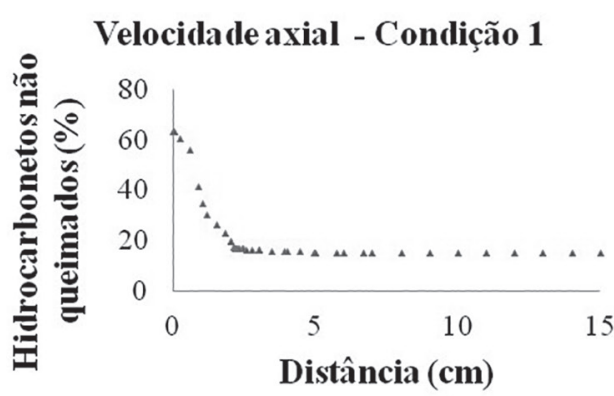

c)

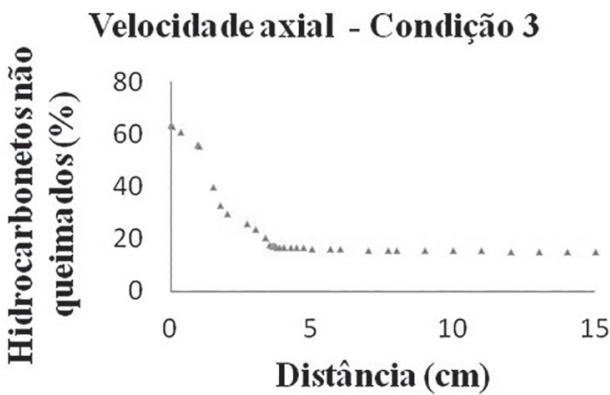

e)

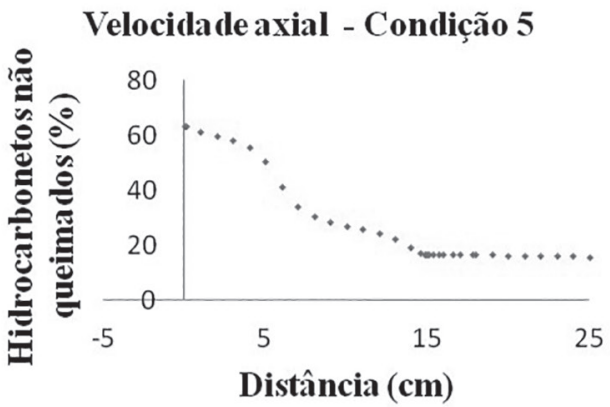

b)

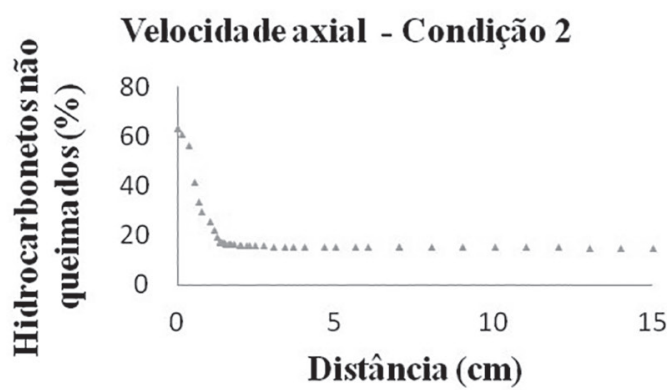

d)

Velocida de axial - Condição 4

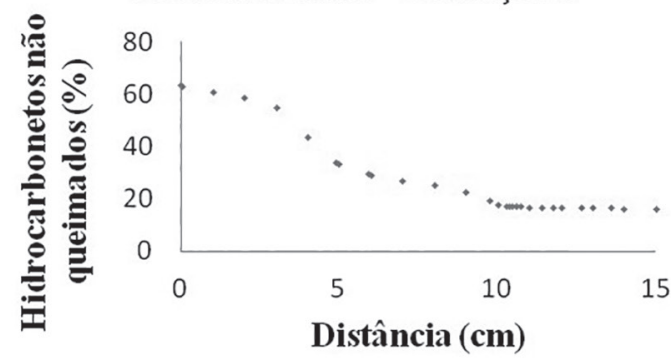

f)

Velocidade axial - Condição 6

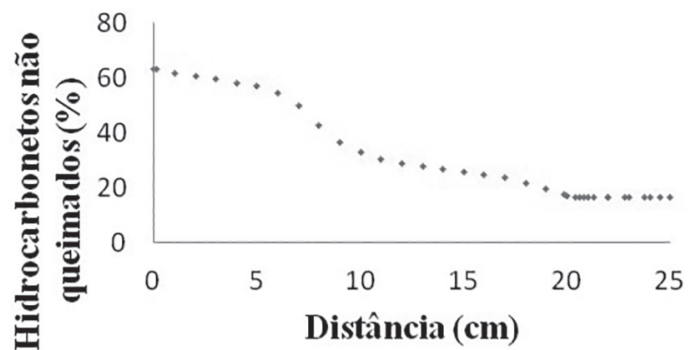

Figura 5. Perfil comparativo de Hidrocarbonetos não queimados em função da distância em diferentes velocidades axiais. a) Condição 1, b) Condição 2, c) Condição 3, d) Condição 4, e) Condição 5, f) Condição 6 (Tabela 3)

onde a distância da região fria foi menor, de $5 \mathrm{~cm}$. Em outras palavras, a distância menor é favorável na queima do propelente pois assim garante a formação dos produtos de combustão ainda no início, de tal forma que ocorra em todo o propelente. Esse efeito da influência da velocidade axial na velocidade da reação também pode ser observado na Figura 5. A Figuras 5a e 5b obtiveram os melhores perfis em relação à velocidade da reação, pois na distância de aproximadamente $5 \mathrm{~cm}$ do propelente, já foi possível notar a formação de produtos de combustão, enquanto que em velocidades axiais maiores, há uma maior região "fria" (temperaturas mais baixas) e consequentemente há uma diminuição na velocidade da reação. Em todas as curvas da Figura 5 obteve-se um resultado de aproximadamente $15 \%$ de hidrocarbonetos não queimados. A Figura 6 apresenta um gráfico 3D com resultados de temperatura máxima pela distância, e a Figura 7 apresenta um gráfico 3D com resultados da fração molar de hidrocarbonetos não queimados pela distância em todas as faixas de velocidade axial estudadas. Pelas Figuras 6 e 7 é possível visualizar os efeitos do aumento da região fria da chama com o aumento da velocidade, bem como o decaimento da velocidade da reação com o aumento da temperatura. Os resultados da Figura 5 estão em $\%$ de hidrocarbonetos não queimados. Entretanto, no gráfico 3D, os resultados estão em ppm devido o software fornecer apenas esse tipo de resultados, não sendo possível converter em \% de hidrocarbonetos não queimados.

No entanto, a escolha da melhor velocidade axial foi realizada após a análise da região "fria" e fração molar dos produtos de combustão incompleta, pois estes são muito poluentes e podem causar danos ao meio ambiente. Mesmo havendo pouca diferença entre as duas condições (Figura 8), optou-se pela velocidade $10^{7} \mathrm{~cm} / \mathrm{s}$, pois foi a velocidade que apresentou menor região "fria" e maior velocidade

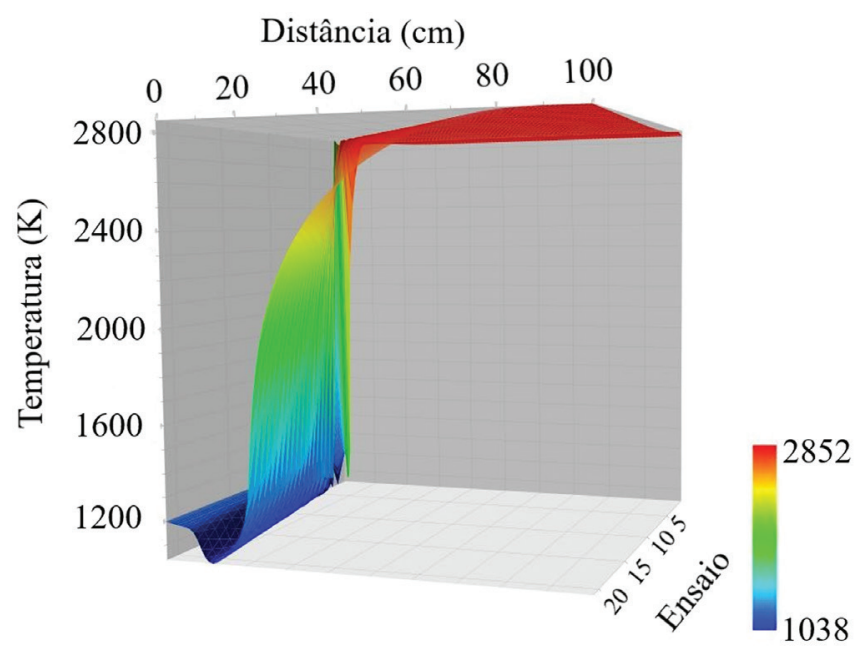

Figura 6. Influência da velocidade axial na temperatura inicial de queima do propelente 


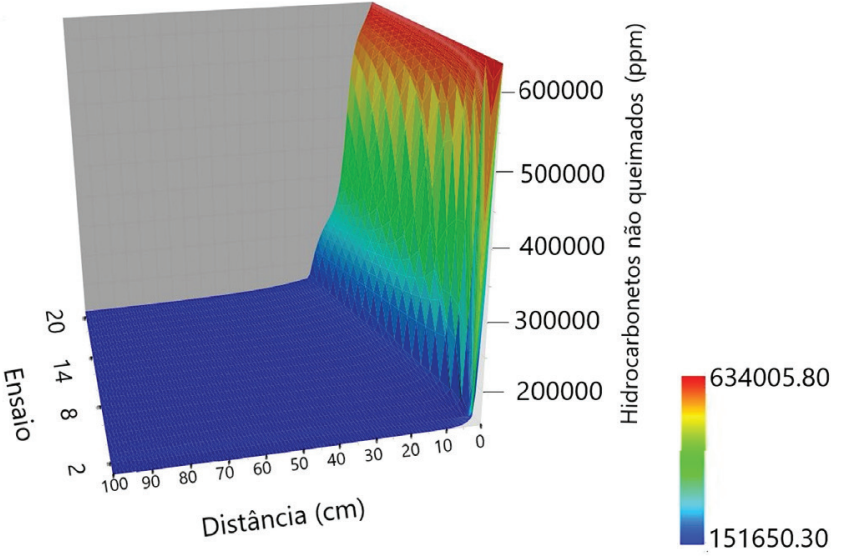

Figura 7. Influência da velocidade axial na velocidade da reação - hidrocarbonetos não queimados

de reação, e além disso, menor quantidade de emissão de poluentes como $\mathrm{CO}, \mathrm{NO}, \mathrm{NO}_{2}$ e $\mathrm{HCl}$. Embora a distância utilizada em todo o processo seja igual à $100 \mathrm{~cm}$, as distâncias utilizadas na Figura 8 foi até $20 \mathrm{~cm}$ para melhor visualização dos produtos de combustão formados.

\section{a) Velocidade axial $10^{6} \mathrm{~cm} / \mathrm{s}$}

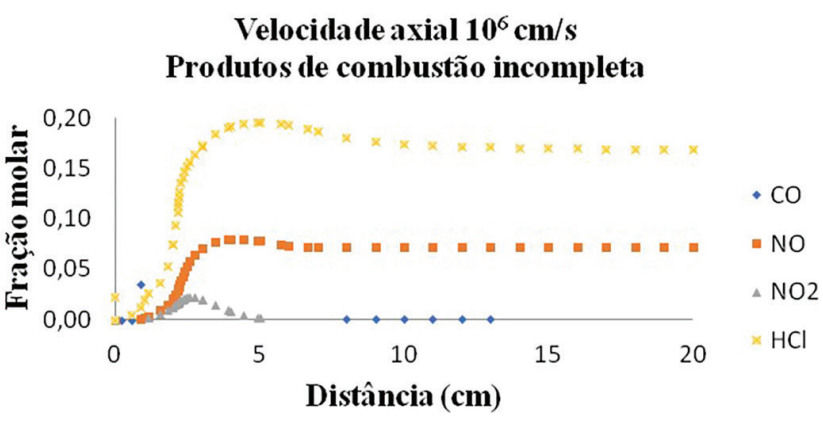

b) Velocidade axial $10^{7} \mathrm{~cm} / \mathrm{s}$

Velocidade axial $10^{7} \mathrm{~cm} / \mathrm{s}$ Produtos de combustão incompleta

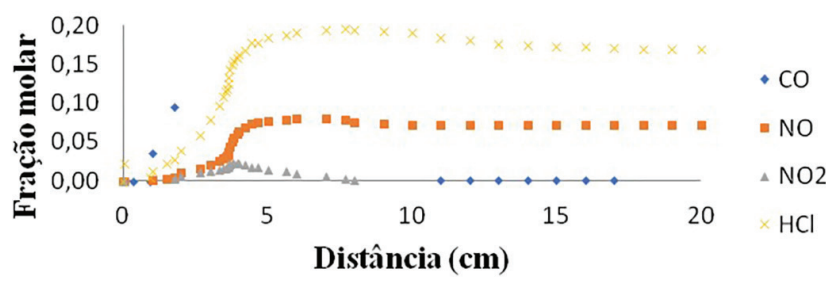

Figura 8. Comparação entre os melhores perfis de Velocidade axial e os produtos de combustão incompleta: a) Velocidade axial $\left.10^{6} \mathrm{~cm} / \mathrm{s} ; b\right)$ Velocidade axial $10^{7} \mathrm{~cm} / \mathrm{s}$

\section{Estudo 2 - Efeito da razão de equivalência}

Nesse estudo analisou-se a influência da quantidade de combustível (matriz polimérica), oxidante (perclorato de amônio) e alumínio na composição do propelente. A razão de equivalência, neste caso, leva em conta que o perclorato de amônio é a espécie oxidante, e os outros componentes da mistura representam o combustível. As razões de equivalência estudadas estão nas Tabelas 2 e 3. Os resultados obtidos se encontram na Tabela 4.

Através dos resultados, foi possível observar que com o aumento da razão de equivalência, houve uma diminuição na temperatura máxima de queima e também uma diminuição na pressão. Como já era esperado, houve um aumento de quantidade de hidrocarbonetos não queimados com o aumento da razão de equivalência. Embora a temperatura, pressão e quantidade de hidrocarbonetos formados sejam relevantes, a razão de equivalência escolhida para seguir o estudo foi a 1, pois esta é a razão de equivalência mais utilizada em propelentes. Além disso, utilizando a razão de equivalência igual a 1, é uma maior certeza que haverá uma combustão mais completa do que aquela utilizada em valores mais baixos.

\section{Estudo 3 - Efeito da pressão}

Para avaliar os efeitos da pressão, realizou-se um estudo em diferentes valores deste parâmetro, utilizando as condições determinadas anteriormente, conforme a Tabela 2 e 3 . Entretanto, não foi possível obter resultados confiáveis pois o reator PFR obtém resultados confiáveis somente em pressões baixas. Em pressões a partir de $10 \mathrm{~atm}$, os resultados obtidos de pressão máxima ficam superiores a 280 atm, o que fica muito fora da faixa de trabalho, pois o propelente sólido compósito apresenta uma pressão de trabalho média de $65 \mathrm{~atm}$. Portanto, para seguir os estudos, a pressão escolhida foi a de $1 \mathrm{~atm}$. A Figura 9 apresenta os resultados obtidos com a variação da pressão.

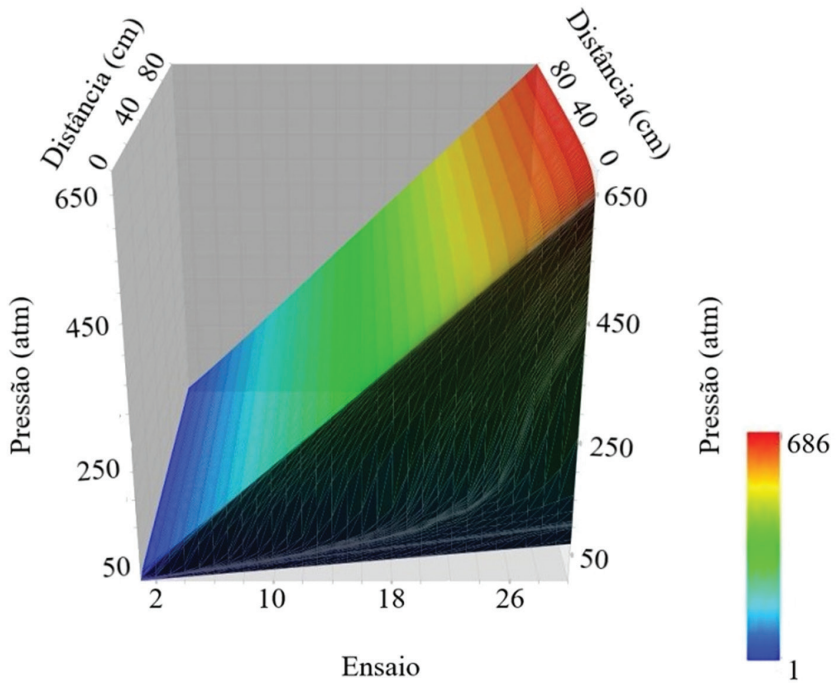

Figura 9. Efeito do aumento da pressão inicial em todas as faixas estudadas

Tabela 4. Resultados da simulação com diferentes razões estequiométricas

\begin{tabular}{ccccc}
\hline Razão Estequiométrica & Temperatura máxima $(\mathrm{K})$ & Pressão $(\mathrm{atm})$ & Hidrocarbonetos não queimados $(\%)$ & Distância percorrida $(\mathrm{cm})$ \\
\hline 0,05 & 2994 & 9,664 & 0,76 & 100 \\
0,25 & 2958 & 9,493 & 3,80 & 100 \\
0,50 & 2913 & 9,289 & 7,60 & 100 \\
0,75 & 2870 & 9,093 & 11,39 & 100 \\
1,0 & 2828 & 8,906 & 15,17 & 100 \\
1,4 & 2794 & 8,761 & 18,17 & 100 \\
\hline
\end{tabular}


Tabela 5. Resultados da simulação em diferentes temperaturas iniciais

\begin{tabular}{ccccc}
\hline Temperatura inicial do gás $(\mathrm{K})$ & Temperatura máxima (K) & Pressão (atm) & Hidrocarbonetos não queimados $(\%)$ & Distância percorrida $(\mathrm{cm})$ \\
\hline 1200 & 2828 & 8,90 & 15,2 & 100 \\
1378 & 2921 & 8,08 & 15,0 & 100 \\
1467 & 2955 & 7,70 & 14,9 & 100 \\
1644 & 3033 & 7,13 & 14,7 & 100 \\
1822 & 3107 & 6,66 & 14,4 & 100 \\
2000 & 3175 & 6,28 & 14,0 & 100 \\
\hline
\end{tabular}

\section{Estudo 4 - Efeito da temperatura}

Para estudar o efeito do aumento da temperatura, variou-se a temperatura inicial do gás, de acordo com as Tabelas 2 e 3 . Os resultados encontram-se na Tabela 5.

Com o aumento da temperatura inicial, houve um aumento da temperatura máxima de queima do propelente, diminuição da pressão máxima e, como esperado, uma diminuição na quantidade de hidrocarbonetos não queimados. Entretanto, em nenhuma das temperaturas utilizadas obteve-se uma pressão próxima à pressão de trabalho do propelente.

\section{Escolha das variáveis utilizando a modelagem no Chemkin}

Para realizar estudos no Chemkin, é necessário definir algumas variáveis iniciais, como por exemplo, a velocidade axial, razão de equivalência, pressão e temperatura inicial de queima. A primeira variável estudada foi a velocidade axial e utilizou-se um estudo paramétrico deste parâmetro. Para avaliar a velocidade axial mais adequada para seguir nos estudos, avaliou-se a menor "região fria" e quantidade de hidrocarbonetos não queimados obtidas em cada velocidade estudada. A velocidade axial escolhida para este estudo foi a $10^{7} \mathrm{~cm} / \mathrm{s}$ pois foi a que obteve a menor "região fria", garantindo a formação dos produtos ainda no início da queima. Dessa forma, há uma maior probabilidade de queima do propelente ainda no início, formando os produtos.

Após a definição da velocidade axial, foi realizado um estudo paramétrico da razão de equivalência. Embora tenha sido possível visualizar a diminuição da temperatura máxima da queima com o aumento da razão de equivalência, a razão de equivalência escolhida para seguir nos estudos foi a 1. Afinal, essa é a razão de equivalência mais comumente utilizada em formulações práticas. Após a determinação da razão de equivalência, e seguindo o estudo paramétrico com o parâmetro de pressão, foi possível observar valores muito superiores comparados aos resultados esperados, que em resultados experimentais ficam na faixa de 55 à 70 atm. Embora a pressão seja um parâmetro muito importante neste estudo, foi possível observar que o reator do programa Chemkin-II utilizado na simulação, o PFR, não apresenta resultados confiáveis em pressões altas. Sendo assim, a pressão escolhida para seguir no estudo foi igual a $1 \mathrm{~atm}$.

Para finalizar, após definir as três condições iniciais, realizou-se um estudo paramétrico da temperatura inicial de queima. Entretanto, embora tenha sido possível observar que com o aumento da temperatura houve uma diminuição dos hidrocarbonetos não queimados e um aumento da temperatura máxima atingida durante a queima do propelente, não foi possível determinar a pressão máxima obtida com essa formulação nas condições pré-determinadas a partir dos estudos anteriores. Isso também pode ser justificado pelo tipo de reator utilizado na simulação. Afinal, em nenhuma temperatura obteve-se um perfil de pressão máxima esperado, que é entre 55 e $70 \mathrm{~atm}$, pois esse reator só é preciso em pressões em torno de
1 atm. Porém, a condição poderia ser determinada de acordo com a temperatura máxima obtida com o propelente, que deve ser em torno de $3000 \mathrm{~K}$, menor quantidade de hidrocarbonetos não queimados, maior quantidade de produtos de combustão completa, menor quantidade de produtos poluentes e pressão máxima próxima à obtida experimentalmente.

\section{CONCLUSÃO}

A escolha das condições otimizadas para a queima do propelente foram realizadas de acordo com o perfil de temperatura de queima, perfil da chama, a partir dos quais foram avaliados a presença de regiões frias, velocidade de reação, quantidade de substâncias poluentes, pressão máxima e resíduos de hidrocarbonetos não queimados.

O software Chemkin pode ser utilizado para simular a queima de formulações de propelente sólido compósito. Embora o reator, chamado PFR do software Chemkin-II, que foi utilizado para as simulações não possa ser utilizado em todas as faixas de pressão, pode-se ter uma ideia do comportamento da queima do propelente e a influência dos parâmetros sobre o comportamento da queima. Os resultados obtidos, como a temperatura máxima do propelente, ficaram muito próximas dos valores encontrados experimentalmente, pois esse tipo de propelente atinge temperatura de cerca de 3000 K. Entretanto, a pressão de trabalho não ficou próxima dos valores reais, que é na média de $65 \mathrm{~atm}$. Isso pode ser explicado devido ao software Chemkin-II utilizado para realizar as simulações realizar os cálculos com pressões de trabalho baixas e, quando se trabalha com pressões muito altas como a do propelente, o software não reproduz resultados muito confiáveis. Contudo, é uma ótima ferramenta para avaliar a influência dos parâmetros.

\section{AGRADECIMENTOS}

À CAPES, ao CNPq e à FAPESP pelos apoios financeiros concedidos.

\section{REFERÊNCIAS}

1. Villar, L.; Silva.R; Rezende,L.; Anais do $10^{\circ}$ Congresso Brasileiro de Polímeros, Foz do Iguaçu, Brasil, 2009.

2. Davenas, A.; Solid Rocket Propulsion Technology, Pergamon: Oxford, 1993.

3. Magalhães, J. B.; Dissertação de mestrado, Instituto Tecnológico de Aeronáutica, Brasil, 2011.

4. Palmerio, A. F.; Introdução à Tecnologia de Foguetes, $2^{\mathrm{a}}$ ed., SindCT: São José dos Campos, 2017.

5. Gonçalves, R.; Ilha, K.; Machado, F.; Rocco, J.; J. Aerosp. Technol. Manage. 2012, 4.

6. Sciamareli, J.; Takashi, K. F. M.; Teixeira, J. M.; Iha, K.; Quim. Nova 2002, 25, 107.

7. Clemente, M. Rocha, R., Iha, K. e Rocco, J.; Quim. Nova 2014, 37, 982. 
8. Nagamachi, M. Y.; Oliveira, J. I. S.; Kawamoto, A. M.; Dutra, R. C. L.; J. Aerosp. Technol. Manage. 2009, 1, 153.

9. Dilsiz, N., Ünver, A.; J. Polym. Sci. 2006, 101, 2538.

10. Kubota, N.; Propellants and Explosives - Thermochemical Aspects of Combustion, $2^{\text {nd }}$ ed., Wiley: Hoboken, 2007.

11. Vilar, W.; Química e Tecnologia de Poliuretanos, $3^{\text {a }}$ ed., Vilar consultoria: Rio de Janeiro, 2004.

12. Cardoso, K. P.; Ferrão, L. F. A.; Kawachi, E. Y.; Araújo, T. B.; Nunes, R. F.; J. Propul. Power 2016, 1.

13. Agnelli, J. A. M.; Tese de Doutorado, Universidade Federal do Rio de Janeiro, Brasil, 1983.

14. Cai, W.; Thakre, P.; Yang, V.; Combust. Sci. Technol. 2008, 180, 2143.

15. Chen, C.-C.; McQuaid M. J.; Proceedings of the $35^{\text {th }}$ JANNAF Propellant and Explosives Characterization Subcommittee Meeting; Las Vegas, USA, 2009.
16. Davidson, J. E.; How to use Chemkin-II, 1996

17. Cochet, J.; Tese de mestrado, University of Melbourne, Australia, 2013.

18. Kee, R. J.; Coltrin, M. E.; Glarborg, P.; Chemically Reacting Flow: Theory and Practice, $1^{\text {st }}$ ed., Wiley Interscience: Hoboken, 2003.

19. Kee, R. J.; Rupley, F. M.; Miller, J. A.; Coltrin, M. E.; Grcar, J. F.; Meeks, E.; Moffat, H. K.; Lutz, A. E.; Dixon-Lewis, G.; Smooke, M. D.; Warnatz, J.; Evans, G. H.; Larson, R. S.; Mitchell, R. E.; Petzold, L. R.; Reynolds, W. C.; Caracotsios, M.; Stewart, W. E.; Glarborg, P.; Wang, C.; Adigun, O.; CHEMKIN Collection, Release 3.6, Reaction Design, Inc., San Diego, CA, 2000. 